\title{
Specific epigenetic alterations of IGF2-H19 locus in spermatozoa from infertile men
}

\author{
Céline Chalas Boissonnas ${ }^{1,2,3}$, Hafida El Abdalaoui ${ }^{4}$, Virginie Haelewyn ${ }^{1}$, Patricia Fauque ${ }^{1,2,3}$, \\ Jean Michel Dupont ${ }^{2,3,5}$, Ivo Gut ${ }^{4}$, Daniel Vaiman ${ }^{2,3}$, Pierre Jouannet ${ }^{1}$, Jörg Tost $^{4}$ and Hélène Jammes ${ }^{\star, 2,3,6}$
}

DNA methylation marks, a key modification of imprinting, are erased in primordial germ cells and sex specifically re-established during gametogenesis. Abnormal epigenetic programming has been proposed as a possible mechanism compromising male fertility. We analysed by pyrosequencing the DNA methylation status of $47 \mathrm{CpGs}$ located in differentially methylated regions (DMRs), the DMRO and DMR2 of the IGF2 gene and in the 3rd and 6th CTCF-binding sites of the H19 DMR in human sperm from men with normal semen and patients with teratozoospermia (T) and/or oligo-astheno-teratozoospermia (OAT). All normal semen samples presented the expected high global methylation level for all CpGs analysed. In the teratozoospermia group, 11 of 19 patients presented a loss of methylation at variable CpG positions either in the IGF2 DMR2 or in both the IGF2 DMR2 and the 6th CTCF of the H19 DMR. In the OAT group, 16 of 22 patients presented a severe loss of methylation of the 6th CTCF, closely correlated with sperm concentration. The methylation state of DMRO and of the 3rd CTCF was never affected by the pathological status of sperm samples. This study demonstrates that epigenetic perturbations of the 6th CTCF site of the $H 19$ DMR might be a relevant biomarker for quantitative defects of spermatogenesis in humans. Moreover, we defined a methylation threshold sustaining the classification of patients in two groups, unmethylated and methylated. Using this new classification of patients, the observed intrinsic imprinting defects of spermatozoa appear not to impair significantly the outcome of assisted reproductive technologies.

European Journal of Human Genetics (2010) 18, 73-80; doi:10.1038/ejhg.2009.117; published online 8 July 2009

Keywords: genomic imprinting; IGF2-H19 locus; methylation; pyrosequencing; spermatozoa; assisted reproductive technologies (ART)

\section{INTRODUCTION}

Genomic imprinting refers to the allele-specific expression of genes in a parent-of-origin specific manner. The most studied molecular modification of imprinting is the differential allele-specific methylation of CpG dinucleotides within differentially methylated regions (DMRs). ${ }^{1}$ Imprinting is early erased in the primordial germ cells ${ }^{2,3}$ and new methylation imprints are established according to the sex of the germ line. ${ }^{1}$ In male mice, $H 19$ and Igf2 methylation is predominantly initiated during the prospermatogonia stage and finalised around the time of birth. ${ }^{4}$ In humans, fetal spermatogonia seem to be mostly unmethylated at the H19 DMR, although spermatogonia of adult testis demonstrate significant methylation in this region. ${ }^{5}$

Aberrant DNA methylation in spermatozoa was previously reported in infertile men. ${ }^{6-8}$ Loss of methylation was detected in the paternally imprinted and methylated DMRs of H19 and GTL2, whereas the maternally imprinted and unmethylated DMR of MEST/PEG1 showed increased methylation for patients with oligospermia, suggesting a simultaneous hypo- and hyper-methylation of the haploid spermatozoa genome. The human IGF2-H19 locus contains three DMRs: the IGF2 DMR0, the IGF2 DMR2 and the H19 DMR (Figure 1a). In this study, we analysed the methylation status of $47 \mathrm{CpGs}$ located in the
DMR0 (3 CpGs) and DMR2 (17 CpGs) of the IGF2 gene and in the 3rd (11 CpGs) and 6th (16 CpGs) CTCF-binding sites of the H19 DMR in human sperm by pyrosequencing to determine the extent and specificity of a potential loss of DNA methylation associated with different sperm abnormalities. Pyrosequencing provides an excellent quantitative resolution of CpG methylation. ${ }^{9}$ The methylation status was analysed in groups of patients with either only severe sperm morphological abnormalities (teratozoospermia), or with associated sperm morphology, mobility and concentration defects (oligoastheno-teratozoospermia) and compared with the methylation patterns of men with normal sperm. Quantitative disruption of the DNA methylation state was correlated with the quantitative semen parameters of all analysed patients. We established a methylation threshold for all analysed CpGs used as a reference and subdividing the patients in two groups, methylated and unmethylated. In both groups, several patients were treated by in vitro fertilization (IVF) or Intra Cytoplasmic Sperm Injection (ICSI) whose outcomes were analysed according to the methylation level of the sperm. To measure the extent of DNA methylation aberrations in OAT patients, we further analysed two maternally methylated DMRs (PEG3 - 14 CpGs and IGF2R - 15 CpGs) as well as four CpGs in the consensus

${ }^{1}$ Biology of Reproduction-CECOS, Cochin-Saint Vincent de Paul Hospital, AP-HP, University Paris-Descartes, Paris, France; ${ }^{2}$ Department of Genetics and Development, Cochin Institute, University Paris-Descartes, Paris, France; ${ }^{3}$ Inserm, U567, Paris, France; ${ }^{4}$ CEA-Institut de Génomique, Centre National de Génotypage, Laboratory for Epigenetics, Evry, France; ${ }^{5}$ Department of Cytogenetics, Cochin-Saint Vincent de Paul Hospital, AP-HP, University Paris-Descartes, Paris, France; ${ }^{6}$ Biology of Development and Reproduction, Institut National de Recherche Agronomique UMR 1198, Jouy en Josas, France

${ }^{*}$ Correspondence: Dr H Jammes, Biology of Development and Reproduction, Institut National de Recherche Agronomique, Domaine de Vilvert, 78352 Jouy en Josas Cedex, France.

Tel: +33 1346521 08, Fax: +33 1346526 77, E-mail: helene.jammes@jouy.inra.fr

Received 24 November 2008; revised 19 May 2009; accepted 22 May 2009; published online 8 July 2009 

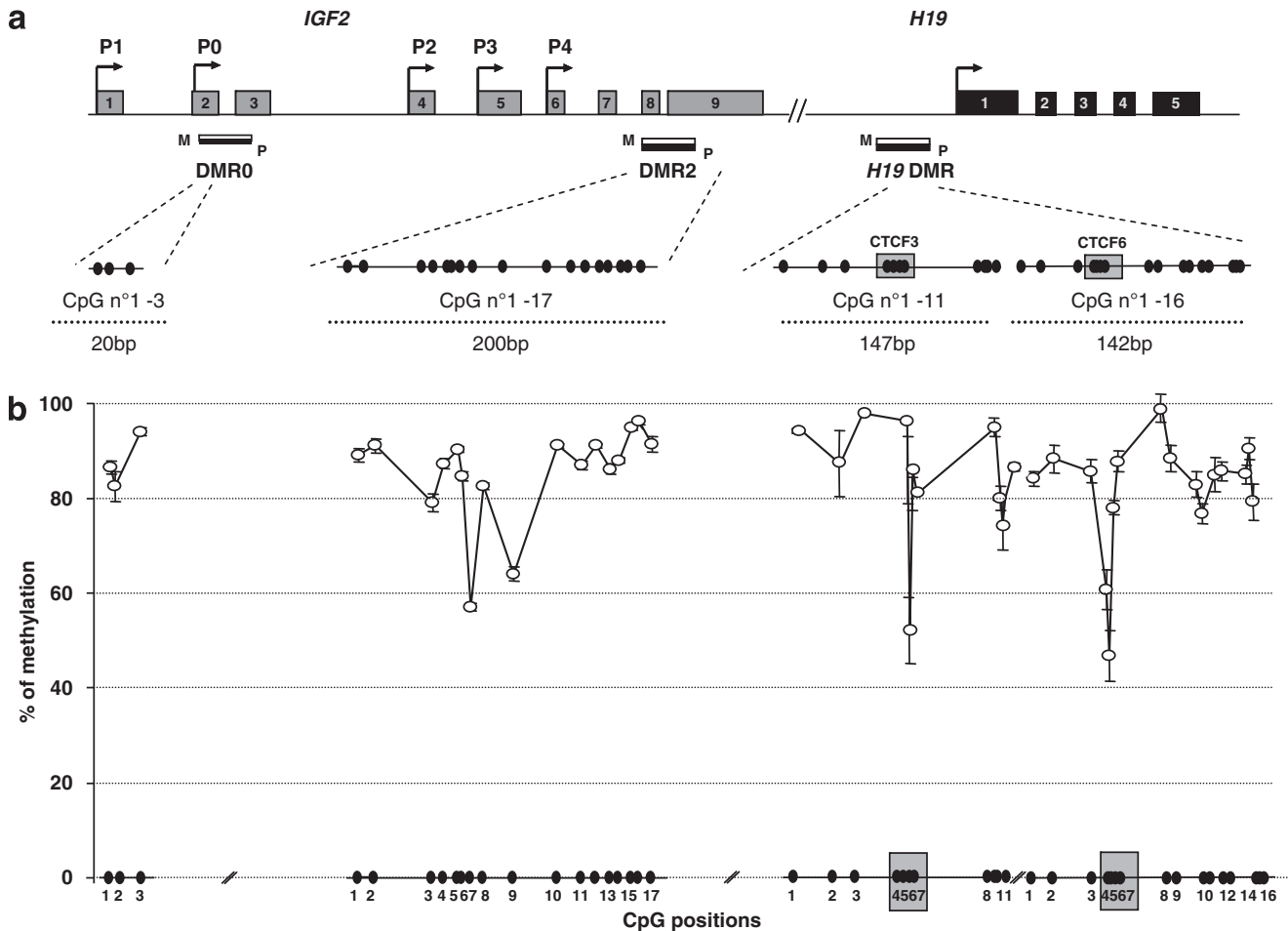

Figure 1 (a) Structural characteristics of the human IGF2-H19 locus. The IGF2 and H19 genes contain 9 exons and 5 exons, respectively (grey and black numbered squares). The transcription start sites and promoters used are indicated by arrows. Regions of differential methylation (DMRs) are shown as black (methylated allele) and white (unmethylated allele) boxes ( $\mathrm{P}=$ paternal, $\mathrm{M}=$ maternal). For the IGF2 gene, two regions were analysed: IGF2 DMRO (3 CpGs) and IGF2 DMR2 (17 CpGs). For the H19 DMR, two of the seven CTCF binding sites and their surrounding CpGs are analysed: the 3rd CTCF (11CpGs) and the 6th CTCF (16 CpGs) binding sites. CpGs corresponding to the two CTCF-binding sites are highlighted by grey squares. For each DMR, the number of CpGs analysed, their relative positions (filled circles) and the length of the amplified sequence in base pairs (bp) are indicated. (b) Methylation pattern at the IGF2-H19 locus in normozoospermic patients (NZ, $n=17$ ). The methylation at each CpG position is expressed in percentage as mean \pm standard deviation. The CpGs 4-7 of the 3rd and of the 6th CTCF-binding site of the H19 DMR are highlighted.

Table 1 Sperm characteristics of the analysed patient cohort

\begin{tabular}{|c|c|c|c|c|c|}
\hline Patient groups & Number of patients & Concentration $10^{6}$ per $\mathrm{ml}$ & Motility (\%) & Vitality (\%) & Typical forms (\%, \\
\hline Teratozoospermia group & 19 & $164.6 \pm 50^{\mathrm{a}}$ & $38 \pm 1.8^{\mathrm{a}}$ & $72 \pm 2.1^{\mathrm{a}}$ & $8 \pm 1.1^{\mathrm{b}}$ \\
\hline OAT-II & 3 & $7.9 \pm 2.2^{b}$ & $30 \pm 14^{a}$ & $73 \pm 11^{a}$ & $6 \pm 5^{b}$ \\
\hline OAT-III & 6 & $3 \pm 1.2^{\mathrm{b}}$ & $20 \pm 14^{b}$ & $58 \pm 15^{b}$ & $12 \pm 14^{b}$ \\
\hline OAT-IV & 6 & $0.17 \pm 0.2^{b}$ & $9 \pm 9^{b}$ & $59 \pm 19^{b}$ & $2 \pm 2^{b}$ \\
\hline
\end{tabular}

For each parameter, the mean values with different letters in exposit position (a,b) are considered significantly different between two groups $(P<0.05)$. The Oligo-astheno-teratozoospermia group (OAT) was subdivised as a function of the sperm concentration (see Materials and methods).

sequences of the LINE1 element as a surrogate for global DNA methylation changes.

\section{PATIENTS AND METHODS}

\section{Patients}

Semen samples were obtained from 58 men (Table 1) aged $36.6 \pm 5.7$ years without statistical age difference between the different patient groups. The first group included 17 men with normal semen parameters (normozoospermia group (NZ), no. 1-17) according to World Health Organization's criteria; ${ }^{10}$ the second group consisted of 19 men presenting normal sperm concentration and $<20 \%$ typical spermatozoa according to David's morphology classification ${ }^{11}$ (teratozoospermia group (T), no. 18-36); the third group included 22 men presenting an oligozoospermia and/or asthenozoospermia and/or teratozoospermia (OAT, no. 37-58). The OAT group was further subdivided as a function of the sperm concentration: OAT-I, $(n=7)$ had a normal sperm concentration (sc $>20 \times 10^{6}$ per $\mathrm{ml}$ ) associated to asthenozoospermia ( $<25 \%$ motile sperm) in three men and to teratozoospermia in four men. OAT-II (sc: $5-10 \times 10^{6}$ per $\mathrm{ml}, n=3$ ), OAT-III (sc: $1-5 \times 10^{6}$ per $\mathrm{ml}, n=6$ ) and OAT-IV ( $\mathrm{sc}<1 \times 10^{6}$ per ml, $n=6$ ) had an increasing severity of oligozoospermia associated to asthenozoospermia and/or teratozoospermia (see Table 1). IVF or ICSI was performed for 24 patients: seven from the NZ group, five from the teratozoospermia group and 12 from the OAT group. All patients gave an informed consent according to the French regulation.

\section{Methods}

Sperm preparation. After 3-5 days of abstinence, ejaculated semen samples were collected at the laboratory and analysed according to WHO recommendations. ${ }^{10}$ Spermatozoa were subsequently selected by centrifugation for $10 \mathrm{~min}$ 
at $600 \mathrm{~g}$ using a Percoll gradient with three concentration layers $(90,60$ and $45 \%$, PureSperm, JCD, Paris, France). The absence of leukocytes and other cells was confirmed by phase contrast microscopic analysis of sperm pellets. Spermatozoa isolated from each sperm pellet sample were subjected to DNA extraction.

DNA extraction. DNA extraction for each individual sample was performed according to the protocol described by Marques $e t a l^{6}$ Briefly, the sperm pellets were overlaid with $100 \mu \mathrm{l}$ of lysis solution (10 mM Tris-HCl, $25 \mathrm{~mm}$ EDTA, 1\% SDS, $75 \mathrm{~mm} \mathrm{NaCl}$ ) in the presence of $0.2 \mathrm{mg} / \mathrm{ml}$ proteinase $\mathrm{K}, 50 \mathrm{~mm}$ DTT and $0.5 \mu \mathrm{g}$ glycogen (Invitrogen, Cergy-Pontoise, France) and incubated overnight at $50^{\circ} \mathrm{C}$. DNA was isolated by standard phenol chloroform extraction.

Methylation analysis. Genomic DNA (500 ng-1 $\mu \mathrm{g})$ was treated with sodium bisulphite. ${ }^{9}$ Bisulphite-treated DNA (50 ng) was subsequently used as template for PCR amplification. Accession numbers and nucleotide positions of each DMR, PCR primers and annealing temperatures as well as the size of PCR products and the number of CpGs analysed are given in Table 2. The 3rd and 6th CTCF-binding sites correspond to CpG 4-7 of each analysed region. Particular attention was paid to the choice of primer sets because a large number of SNPs were described in these regions. In particular, CpG 5 of the sixth CTCF-binding site is a C/T SNP (rs 10732516) and this CpG was therefore not considered for the quantitative methylation analysis. Reaction conditions were $1 \times$ HotStar Taq buffer supplemented with $1.6 \mathrm{~mm} \mathrm{MgCl} 2$, $100 \mu \mathrm{m}$ dNTPs, $2.0 \mathrm{U}$ HotStar Taq polymerase (Qiagen, Courtaboeuf, France) and 5 to $7.5 \mathrm{pmol}$ of forward and reverse primers, one of them being biotinylated, in a $25 \mu \mathrm{l}$ volume. The PCR programme consisted of a denaturing step of $15 \mathrm{~min}$ at $95^{\circ} \mathrm{C}$ followed by 50 cycles of $30 \mathrm{~s}$ at $95^{\circ} \mathrm{C}, 30 \mathrm{~s}$ at the respective annealing temperature (Table 2) and $20 \mathrm{~s}$ at $72^{\circ} \mathrm{C}$, with a final extension of $5 \mathrm{~min}$ at $72^{\circ} \mathrm{C}$. Pyrosequencing was done as previously described using the PSQ 96MA system with the PyroGold SQA reagent kit according to the manufacturer's instructions (Pyrosequencing AB, Uppsala, Sweden). ${ }^{9}$ DNA methylation levels were calculated as ratio of the $\mathrm{C}$ to $\mathrm{T}$ peaks at a given $\mathrm{CpG}$ site in pyrograms. From the quantitative results obtained by pyrosequencing in normozoospermic patients, a mean level of methylation was calculated for each CpG position and a methylation threshold was defined as the mean value minus two SD. This threshold was used to analyse the methylation state of the teratozoospermia and OAT patient groups and to classify them as being methylated or unmethylated compared with the control group (with $P<0.05$ ).

Statistical analysis. Student's $t$-test was used throughout this study, with a $P$-value $<0.05$ threshold considered as significant.

\section{RESULTS}

\section{Normozoospermic patients}

A highly specific and reproducible profile of methylation was observed for all normozoospermic patients of the control group displaying the expected high methylation level (Figure 1b). For the IGF2 DMR0, the mean methylation level was found to be $87.7 \pm 5 \%$. This study is the first demonstration of a methylation of the IGF2 DMR0 in human spermatozoa. The IGF2 DMR2 exhibited a very specific methylation pattern with a high methylation level for all CpGs except CpG 7 and 9, which had methylation levels of 58 and $62 \%$, respectively. A similar decrease of these two CpGs has been observed in other human tissues (Jammes H, unpublished) and these two CpGs were excluded for the calculation of the mean methylation level of the IGF2 DMR2 $(88.7 \pm 1.9 \%)$. The amplification products analysing the 3rd and 6th CTCF-binding sites of the H19 DMR exhibited also a high methylation level with a mean DNA methylation level of $87.2 \pm 5.3$ and $83.7 \pm 7.7 \%$, respectively. Particularly, CpGs $4-7$, which are exactly located on the CTCF-binding sites, were highly methylated.

\section{Teratozoospermic patients}

In the sperm from teratozoospermic patients, a high methylation level was observed for the IGF2-DMR0 $(90.4 \pm 2.8 \%)$ without any signifi-

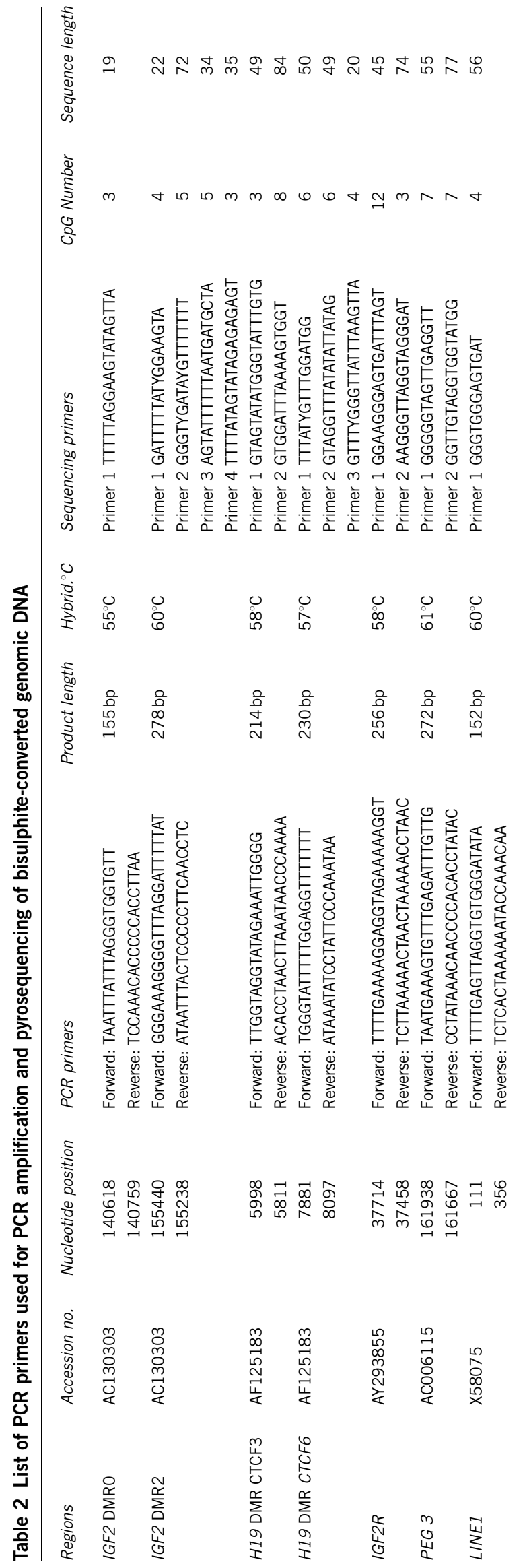

European Journal of Human Genetics 
Table 3 Statistical analysis of the methylation differences at sporadic CpG positions between pathological (teratozoospermic and OAT) and normozoospermic groups.

\begin{tabular}{lccc}
\hline IGF2 DMRO & & & \\
\hline CpG number & CpG 1 & CpG 2 & \\
Teratozoospermia versus control & 0.46 & 0.05 & CpG 3 \\
OAT versus control & 0.62 & 0.29 & 0.46
\end{tabular}

OAT versus control

\section{IGF2 DMR2}

\begin{tabular}{lccccccccccccccccc}
\hline CpG number & CpG1 & CpG2 & CpG3 & CpG4 & CpG5 & CpG6 & CpG7 & CpG8 & CpG9 & CpG10 & CpG11 & CpG12 & CpG13 & CpG14 & CpG15 & CpG16 & CpG17 \\
Teratozoospermia versus control & 0.59 & 0.90 & 0.81 & 0.64 & 0.68 & 0.4 & $\mathbf{0 . 0 3}$ & 0.96 & 0.89 & 0.13 & 0.19 & 0.19 & 0.85 & $\mathbf{0 . 0 1}$ & 0.34 & 0.56 & 0.59 \\
OAT versus control & $\mathbf{0 . 0 4}$ & 0.13 & 0.41 & $\mathbf{0 . 0 1}$ & $\mathbf{0 . 0 0 9}$ & $\mathbf{0 . 0 4}$ & 0.82 & $\mathbf{0 . 0 2}$ & 0.16 & $\mathbf{0 . 0 1}$ & $\mathbf{0 . 1}$ & $\mathbf{0 . 0 5}$ & $\mathbf{0 . 2 2}$ & $\mathbf{0 . 0 1}$ & $\mathbf{0 . 0 4}$ & $\mathbf{0 . 0 1}$ & $\mathbf{0 . 0 7}$
\end{tabular}

H19 CTCF3

\begin{tabular}{lccccccccccc}
\hline CpG number & CpG2 & CpG2 & CpG3 & CpG4 & CpG5 & CpG6 & CpG7 & CpG8 & CpG9 & CpG10 & CpG11 \\
Teratozoospermia versus control & 0.62 & 0.22 & 0.35 & 0.95 & 0.55 & 0.23 & 0.03 & 0.58 & 0.92 & 0.25 & 0.62 \\
OAT versus control & 0.27 & 0.09 & 0.64 & 0.65 & 0.98 & 0.27 & 0.97 & 0.64 & 0.26 & 0.24 & 0.17
\end{tabular}

H19 CTCF6

\begin{tabular}{|c|c|c|c|c|c|c|c|c|c|c|c|c|c|c|c|c|}
\hline CpG & CpG1 & CpG2 & CpG3 & CpG4 & CpG5 & CpG6 & CpG7 & CpG8 & CpG9 & CpG10 & CpG11 & CpG12 & CpG13 & CpG14 & CpG15 & pG16 \\
\hline & 0.08 & 0 & & 0.87 & & & & & .37 & & & & & 0 & .75 & .85 \\
\hline
\end{tabular}

OAT versus control

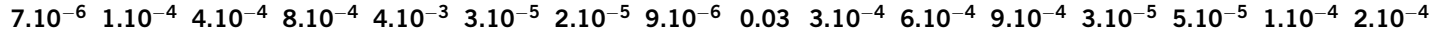

Only the $P$-value is presented. The difference between two methylation values for a CpG position was considered significant when $P<0.05$ (bold characters).

cant difference compared with the control group (Table 3). The IGF2DMR2 was also similarly methylated $(84.4 \pm 3.4 \%)$. The $11 \mathrm{CpGs}$ of the 3rd CTCF-binding site remained at a similar methylation level compared with NZ group (91.4 \pm 2 versus $87.2 \pm 5.3 \%$ ), as was the mean methylation level of the 6th CTCF-binding site $(79 \pm 7.9 \%)$. However, when analysing each CpG position separately, CpG 7 of the 3rd CTCF and CpG 6 and 8 of the 6th CTCF displayed a significant difference in comparison to the control group (Table 3 ). It should be noted that these CpGs showed, in general, a high degree of interindividual variability of their respective methylation levels. Samples from the teratozoospermia group were classified into two groups according to the threshold calculated from the NZ samples. Only eight patients displayed the expected methylation patterns, whereas 11 patients $(58 \%)$ exhibited a significant hypomethylation at different CpG positions (Figure 2a and b). No statistical difference in the type and location of morphological abnormalities (head, mid-piece or flagellar level) of the sperm cells was observed among both the subgroups.

\section{Oligo-astheno-teratozoospermic patients}

In the OAT group, a high methylation level similar to the NZ group was observed for the IGF2 DMR0 $(89.5 \pm 5.4 \%)$ as well as for the 3rd CTCF-binding site in the H19 DMR ( $89.5 \pm 3.6 \%$; Table 3). However, a drastic decrease of the methylation level was observed for the IGF2 DMR2 (OAT versus $\mathrm{NZ}-78 \pm 15.2$ and $88.7 \pm 1.9 \%$, respectively) with a significant difference for 10 of $17 \mathrm{CpG}$ positions (Table 3). The 6th CTCF-binding site in the H19 DMR exhibited a strongly altered methylation level $(53 \pm 26.3$ versus $83.7 \pm 7.7 \%$ in the NZ group, $P<0.01)$ and all CpGs exhibited a significantly lower methylation level (Table 3, Figure 3). Using the methylation thresholds defined in the NZ cohort, 15 samples out of $22(68 \%)$ exhibited a severe loss of methylation (Figure $3 \mathrm{a}$ and $\mathrm{b}$ ).
A normal methylation state was only observed in the OAT-I subgroup with a sperm concentration above $20 \times 10^{6}$ per ml (Figure $3 \mathrm{~b}$; with a methylation mean of $87.2 \pm 6.5 \%$ for DMR0, of $86.6 \pm 4.13 \%$ for DMR2, of $88.5 \pm 3 \%$ for H19 DMR CTCF3 and of $76.7 \pm 3.6$ for H19 DMR CTCF6). The loss of methylation of both IGF2 DMR2 and H19 DMR 6th CTCF was found highly significant for the subgroups OAT-II, OAT-III and IV $(P<0.01)$ corresponding to a sperm concentration below $10 \times 10^{6}$ spermatozoa per ml (Figure $3 \mathrm{~b}$ ). A linear correlation between the mean methylation level of the 6th CTCF-binding site and sperm concentration was observed with a correlation coefficient of $R^{2}=0.61$ (Figure 4).

We also tested if there were possible concomitant gains of methylation at regions that have a maternally acquired methylation imprint. A low methylation level was observed for the PEG3 DMR (14 CpG analysed) in the NZ group as well as in the OAT group (4.6 \pm 0.9 and $5.8 \pm 3 \%$, respectively). An increase in the methylation level was observed for the IGF2R DMR in the OAT group (OAT versus $\mathrm{NZ}-9.1 \pm 3.9$ and $7 \pm 2.5 \%$, respectively) with a significant difference for 6 of $15 \mathrm{CpG}$ positions. To further investigate if sperm samples from OAT patients displayed global DNA methylation changes, we analysed methylation in the LINE1 element as a surrogate. OAT patients displayed a tendency to lower methylation levels $(46 \pm 3.8 \%$ in NZ versus $44 \pm 4.1 \%$ in OAT), although not reaching statistical significance (data not shown).

\section{Assisted reproductive technology (ART) outcomes}

Twenty-four men and their partners were treated by 44 ART cycles to procreate. In vitro fertilization was performed for seven men from the NZ group (14 cycles), whereas ICSI was performed for five men from the teratozoospermia group ( 9 cycles) and 12 men from the OAT group ( 21 cycles). The 24 individuals were classified into two groups: 13 men whose sperm was over the threshold (methylated) with a mean 
a

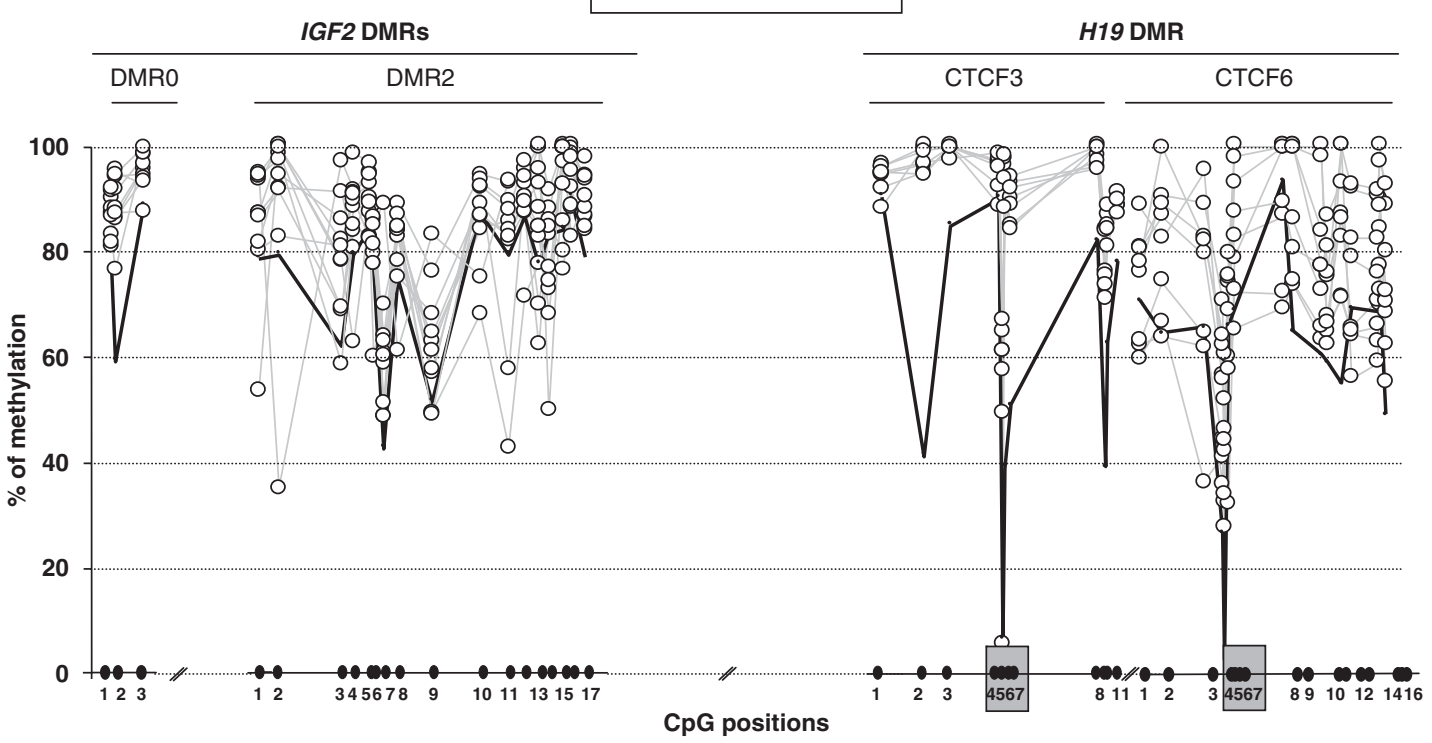

b IGF2 DMR2 CpGs

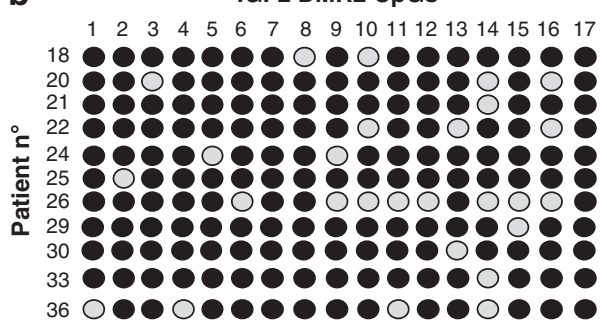

H19 DMR $6^{\text {th }}$ CTCF CpGs

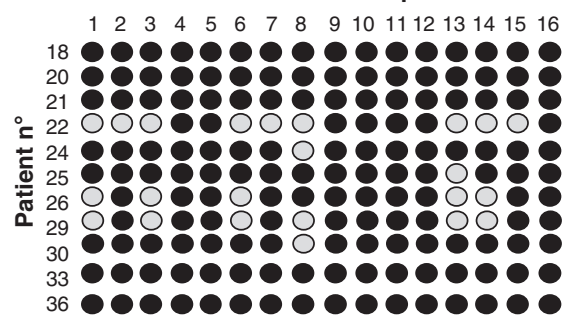

CpG position methylation > methylation threshold

$\bigcirc \mathrm{CpG}$ position methylation $<$ methylation threshold

Figure 2 (a) Methylation pattern at the IGF2-H19 locus in teratozoospermic patients. From the methylation results of the NZ group, a mean level of methylation was calculated for each CpG position. A methylation threshold (black line) is defined as the mean value minus two SD. The methylation threshold delimits a subgroup of teratozoospermic patients $(n=11)$ exhibiting a decrease of the DNA methylation level at specific CpG positions in the IGF2 DMR2 and the 6th CTCF-binding site of the H19 DMR. The CpG positions are represented by open circles and each line represents an individual sample analysis. Only the dots underneath the black line indicate a significant reduction of DNA methylation. (b) Alternative representation for Figure 2a. Using the methylation threshold, the closed circles represent methylated $\mathrm{CpGs}$ while the grey circles represents $\mathrm{CpGs}$ with a methylation level significantly different from the NZ group $(P<0.05)$. CpG positions are indicated vertically (CpG 1-17 for the IGF2 DMR2 and CpGs 1-16 for the 6th CTCF-binding site of the H19 DMR). The patients are numbered and represented in lines. Eleven teratozoospermia patients showed an altered methylation status of CpG positions in the IGF2 DMR2 and 6 of them showed also alterations in the 6th CTCF-binding site of the H19 DMR.

methylation of $88.7 \pm 2.1 \%$ and of $84.3 \pm 7.9 \%$ for the IGF2 DMR2 and the 6th CTCF of the H19 DMR, respectively, and 11 men whose sperm was under the threshold (unmethylated) with a mean methylation of $66.6 \pm 15.8$ and $34.1 \pm 12.1 \%$, for the IGF2 DMR2 and the 6th CTCF of the H19 DMR, respectively. The 13 men of the M group belonged to the $\mathrm{NZ}$ group $(n=7)$, the teratozoospermia group $(n=5)$ and the OAT-I group $(n=1)$ and the 11 men of the UM group had all an sc $<10 \times 10^{6}$ per $\mathrm{ml}$ and were from the OAT-II $(n=1)$, III $(n=4)$ and IV $(n=6)$ groups. The mean fertilization rate was significantly different between both groups $(63.6 \%$ in UM versus $83 \%$ in $M$, $P<0.05)$. No difference could be observed between both groups in early cleavage kinetic as evaluated by the number of blastomers or in embryo fragmentation 2 days after insemination or injection. The implantation rate was similar in both groups (unmethylated $16.3 \%$ versus $M 18.9 \%$ ). The delivery or ongoing pregnancy rate per oocyte pickup was $20 \%$ in the UM group (20 cycles) and $33.3 \%$ in the M group ( 24 cycles) but the difference was not significant. No difference in the delivery term was observed between both groups $(40.3 \pm 0.5$ weeks in UM $(n=4)$ and $40.7 \pm 0.58$ weeks in M $(n=8)$, respectively) as well as for the birth weight of the newborns $(3053 \pm 90 \mathrm{~g}$ in UM and $3090 \pm 334 \mathrm{~g}$ in $\mathrm{M}$, respectively).

\section{DISCUSSION}

Paternal IGF2 and maternal H19 expression are closely regulated by genetic imprinting including numerous regulatory mechanisms such as allele-specific DNA loops, multiple enhancers, boundary elements and histone modifications. ${ }^{12-14}$ The human locus contains three DMRs: the DMR0 and DMR2 of the IGF2 gene and the H19 DMR. A notable discrepancy to the mouse Igf2-H19 locus is the absence of a human IGF2 DMR1. In human sperm from fertile and infertile men, only the methylation status of the 6th CTCF-binding site of the H19 DMR was previously analysed. ${ }^{5-8}$ 

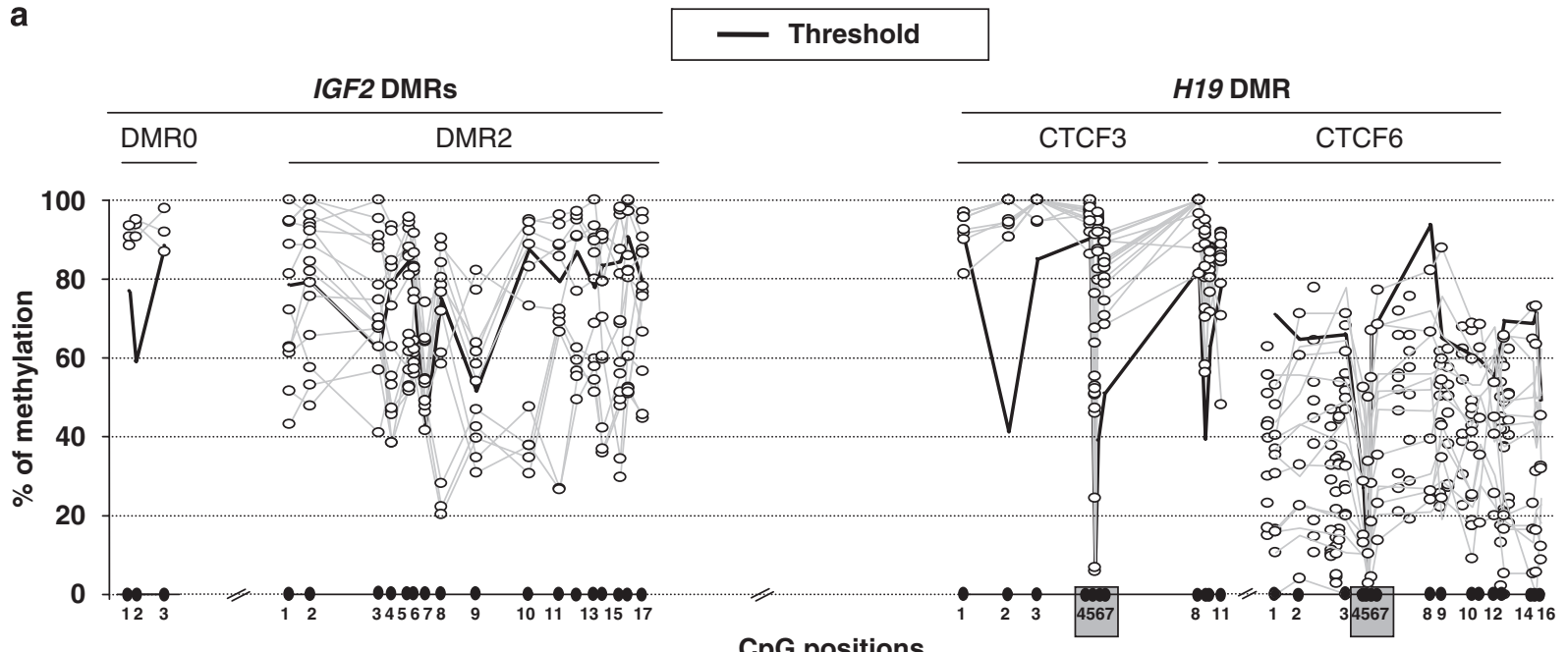

b
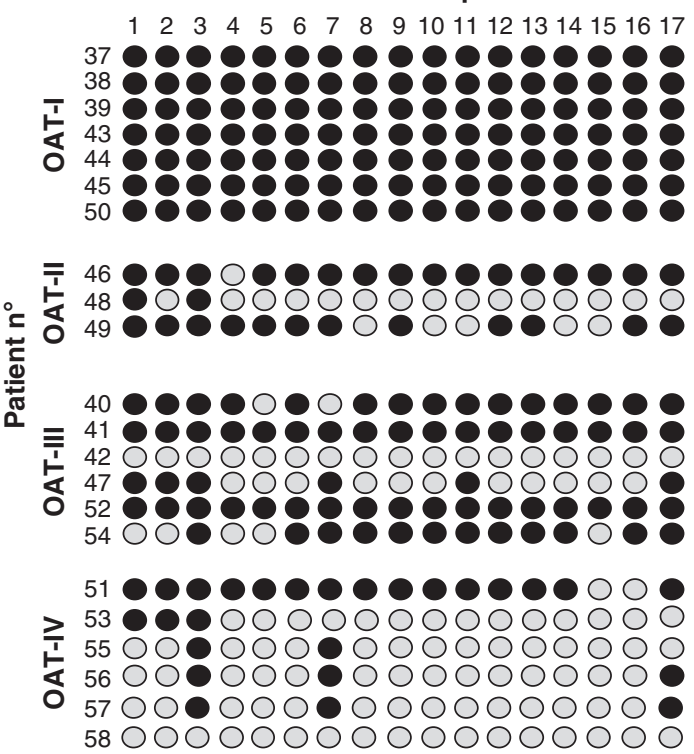

H19 DMR $6^{\text {th }} \mathrm{CTCF}$ CpGs

$\begin{array}{lllllllllllll}1 & 2 & 3 & 4 & 5 & 6 & 7 & 8 & 9 & 10111213141516\end{array}$

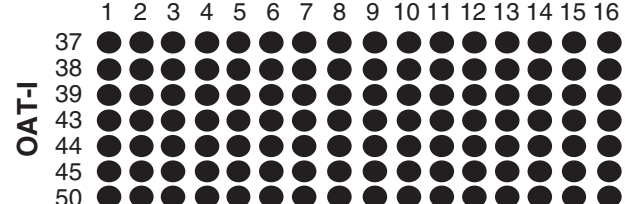

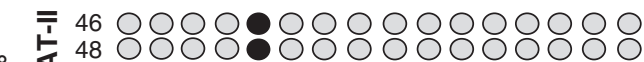
¿ \& 490080800080080000

营

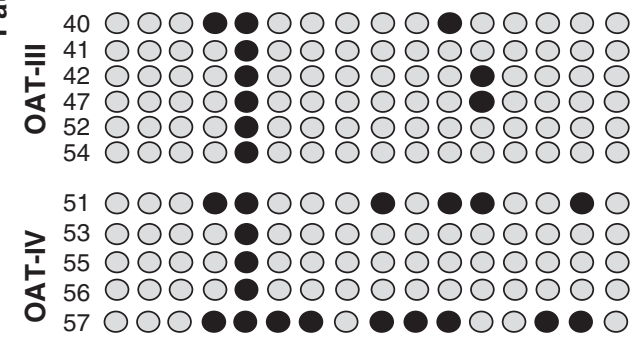

580000000000000000

CpG position methylation > methylation threshold

CpG position methylation < methylation threshold

Figure 3 Methylation pattern of IGF2-H19 locus in oligo-asthenoteratozoospermic patients. (a) The methylation threshold (black line) delimits a subgroup of OAT patients $(n=15)$ exhibiting a drastic decrease of methylation level of H19 DMR (6th CTCF-binding site) associated with a loss of methylation at CpG positions in the IGF2 DMR2. The CpG positions are represented by open circles and each line represents an individual sample analysis. Only the dots underneath the black line indicate a significant reduction of DNA methylation. (b) Alternative representation for Figure 3a using the OAT subgroups as defined by sperm concentration. The closed circles represent methylated $\mathrm{CpGs}$, whereas the grey circles represent $\mathrm{CpGs}$ with a methylation level significantly different from the NZ group $(P<0.05)$. CpG positions are indicated vertically (CpG 1-17 for IGF2 DMR2 and CpGs 1-16 for H19 DMR). The patients are numbered and represented in lines.

In this study, we report for the first time the methylation status of the two IGF2 DMRs (DMR0 and DMR2) and of the 3rd and 6th CTCF-binding sites of the H19 DMR in spermatozoa from men presenting normal and altered sperm parameters. Pyrosequencing is an exceedingly quantitative technology yielding accurate and highly reproducible information about methylation positions at a singlenucleotide resolution. ${ }^{9}$

In normozoospermic patients, a mean methylation level of $86.8 \pm 6.9 \%$ was found for these four regions. Taking into account the inter-assay variation, this suggests either a complete demethylation of $\sim 10 \%$ of spermatozoa in normal human sperm or the existence of $\sim 10 \%$ of unmethylated $\mathrm{CpG}$ at one position or another. In concordance with our results, a low proportion of unmethylated paternal haploid genome in normal human semen was previously reported. ${ }^{6-8}$ Moreover, for specific CpG positions inside the IGF2 DMR2 (CpG 7 and $\mathrm{CpG} 9$ ), the methylation mean varied significantly displaying a specific methylation profile in this region and suggesting that the local chromatin architecture might influence the accessibility to methyl- 


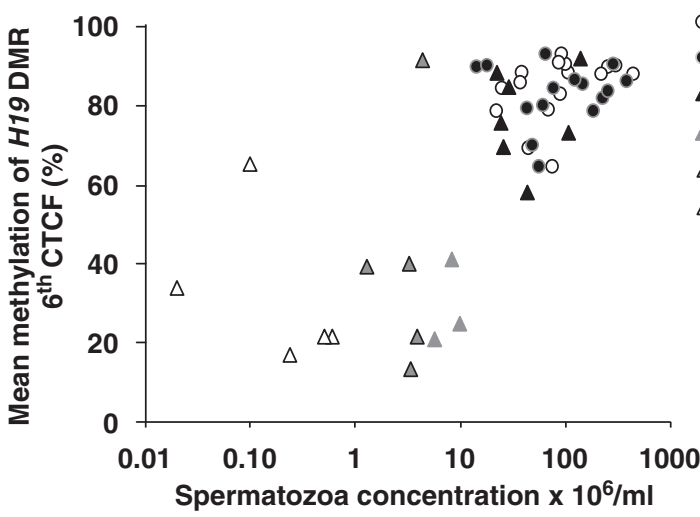

Figure 4 Relationship between the DNA methylation level of the 6th CTCFbinding site in the H19 DMR and sperm concentration. (NZ: normozoospermia group; T: teratozoospermia group; OAT: oligo-asthe noteratozoospermia group).

transferase activity. The systematic microscopic control of the spermatozoa preparation in our study eliminated the possibility of an eventual somatic cell contamination.

In this study, the IGF2 DMR0 was found highly methylated. The DMR0 may have an important role in the regulation of IGF2 imprinting control because this region is hypomethylated and associated with loss of imprinting in Wilms' tumours and colorectal cancers. ${ }^{15-17}$ The mouse Igf2 DMR0 was previously described as only maternally methylated in placenta and with a methylation of the two alleles in other tissues. ${ }^{13,18}$ In humans, the DMR0 is differentially methylated in tissues as blood cells, placenta and kidney. ${ }^{16,19}$ These two studies reported the methylation to be on the maternal allele in the DMR0. Our data provides evidence of a methylation of IGF2 DMR0 in spermatozoa. This is in concordance with the recent report of Murrell et al, ${ }^{20}$ analysing the DMR0 methylation status in growth disorders, cancers and normal tissues and suggesting that the active paternal IGF2 allele is methylated at the DMRO in cis with $\mathrm{H} 19$ methylation in normal individuals. In our study, the methylation pattern of DMR0 was similar in normal and abnormal sperms. The three other regions exhibited the expected DNA methylation profile corresponding to a paternal methylation imprint in normozoospermic patients.

The quantitative pyrosequencing analysis of normal sperm allowed us to define a threshold for the methylation status for each CpG position and to precisely detect sporadic or severe significant methylation defects at individual or consecutive $\mathrm{CpG}$ positions in sperm samples of infertile men.

In the samples with isolated abnormalities of spermatozoa morphology ( $\mathrm{T}$ ), a normal methylation profile of the four regions was only observed for $42 \%$ of patients. Thus, $58 \%$ of the sperm samples presented a significant loss of methylation at particular $\mathrm{CpG}$ positions for the IGF2 DMR2 that was associated in six men with a loss of methylation at some CpG positions in the 6th CTCF-binding site of the H19 DMR. To our knowledge, these methylation modifications were not previously reported in patients with teratozoospermia. No significant association was found between the loss of methylation and any specific morphological abnormality according to accurate and detailed analysis of sperm morphology. ${ }^{11}$

The loss of methylation in the IGF2 DMR2 and the 6th CTCFbinding site of the H19 DMR were accentuated in the samples with low-sperm concentrations (OAT-II to OAT-IV; 59\% of patients). No methylation changes were observed for the IGF2 DMR0 or the 3rd CTCF-binding site, demonstrating that inside a $\sim 100 \mathrm{~kb}$ locus, two regions with abnormal methylation profile and two regions without alterations can exist and highlighting the locus-specificity of the methylation loss associated with spermatogenesis failure.

Previous reports showed an abnormal paternal methylation imprint of the 6th CTCF-binding site of the H19 DMR, as well as GTL2 in a low proportion of patients with moderate and severe oligospermia. ${ }^{6-8}$ The same reports showed also a frequent gain of methylation in regions with a maternal methylation imprint (MEST/PEG1, LIT1, ZAC, PEG3 and SNRPN DMRs). ${ }^{8}$ These results suggest a rise of methylation or a failure in erasure during germ-cell development. Nevertheless, the methylation profile of non-imprinted repetitive elements, such as long interspersed nucleotide elements (LINE1) and $A L U$ element was normal ${ }^{7,8}$ contradicting the reports of increased DNA methylation in poor quality human sperm. ${ }^{21}$ Our results support this hypothesis of strong methylation errors occurring at specific loci in sperm from infertile men without genome-wide alterations.

Although somatic cells were not observed by microscopic inspection of all spermatozoa preparations, a very improbable somatic contamination cannot be fully excluded. The expected methylation status of the somatic cells present in the ejaculate (round cells and leukocytes) is due to paternal methylated and maternal unmethylated alleles at the IGF2/H19 DMRs leading to a 50\% of methylation that would be quantified by pyrosequencing. In our study, the methylation losses are from 10 to $80 \%$. An undetectable somatic cell contamination by microscopy could not account for such a large reduction in methylation level consistent over the analysed subgroups.

The swim-up technique is the best available method for selecting spermatozoa and eliminates somatic cell contamination leading to a selection of highly mobile spermatozoa. Nevertheless, this technique could not be used in our study for preparation of sperm from OAT patients who have a dramatic reduction of the sperm motility. Moreover, the use of highly selected spermatozoa with a good motility would distort the methylation analysis. We therefore applied the described spermatozoa preparation protocol that can be applied to all patient groups.

The observed DNA methylation alterations could be due to failures in chromatin compaction events that are highly controlled by essential sperm-specific chromatin proteins. ${ }^{22}$ The haploid phase of spermatogenesis is marked by the expression of protamines facilitating the molecular remodelling and specific compaction of the male genome associated with gene silencing. However, the human sperm nucleus retains about $15 \%$ of the somatic histones. ${ }^{23}$ The nucleohistone and nucleoprotamine compartments form discrete structures that are at least partly sequence specific. ${ }^{23}$ The telomeric repetitive sequences are histone-rich, whereas the $\beta$-globulin and Alu sequences are protamine-enriched. ${ }^{24}$ The IGF2 gene retains the nucleohistone compartment, whereas the $H 19$ gene is predominantly organised by a unique structural repeat condensing at least $20 \mathrm{~kb}$ of chromatin. ${ }^{25}$ The nucleohistone/nucleoprotamine partition of the sperm genome could support epigenetic information and operate in the imprinting establishment.

The observed alterations might also be related to less efficient DNA methyltransferases, especially DNMT3L and 3A. In mice, the Dnmt3L and conditional Dnmt3A KO result in azoospermia whereas Dnmt3a KO disturbs the methylation imprinting. ${ }^{26,27}$ It would therefore be interesting to measure the Dnmt3A/3L activities in human spermatozoa in case of oligozoospermia. 
As the methylation mark apposition occurs at the spermatogonia stage, ${ }^{5}$ it could be hypothesised that the initial cause of the DNA methylation defects observed for the infertile men is either a failure of the resetting of methylation in a subset of adult spermatogonia or an alteration of the methylation maintenance in spermatocytes, spermatids or mature spermatozoa. Through testicular biopsies, it will be informative to collect spermatogonia, spermatocytes, spermatids and spermatozoa to compare the methylation status of the IGF2-H19 locus in men with or without infertility.

A possible link between ART and imprinting congenital disorders, such as Angelman, Prader-Willi and Beckwith-Wiedemann syndromes has been suggested by several groups. ${ }^{28-30}$ It is so far unclear at which stage(s) the epigenetic alterations could arise during the ART procedures. It has been argued that the use of spermatozoa from men with abnormal semen parameters and abnormal IGF2-H19 methylation status could affect imprinting. Two previous studies reported ART outcomes for men with methylation defect of their sperm: Kobayashi et $a l^{8}$ described a low percentage of pregnancies with several miscarriages in samples presenting abnormal imprinting. Benchaib et $a^{31,32}$ correlated a global sperm hypomethylation and low pregnancy rates without differences in fertilization rates or embryo quality. Using the methylation threshold concept, we could classify the 24 men who benefitted from ART into two groups, methylated and unmethylated. All the UM patients presented an oligozoosspermia, whereas the $\mathrm{M}$ patients had normal sperm characteristics or only abnormal sperm morphology. In our laboratory, no significant difference in rate fertilization was found between IVF and ICSI (64.5 versus 70\%, respectively). Taking this information into account, it could be considered that the fertilization rate is expected in the UM group (63\%) and increased in the $\mathrm{M}$ group (83\%), whereas embryo quality and implantation rate were similar. Normal pregnancies, deliveries at term and birth of healthy newborns were obtained from men whose sperm had a drastic methylation loss. The delivery and ongoing pregnancy rate differed with a higher percentage in the group $\mathrm{M}$ but the difference was not significant. Larger studies are required to obtain sufficient statistical power to fully evaluate these potential associations.

Further studies might also address the relationship between the methylation status and the fertilization ability of individual spermatozoa.

\section{ACKNOWLEDGEMENTS}

Studies in the Biologie de la Reproduction laboratory and CECOS have been supported by INSERM Unité 567, UMR CNRS 8103, Université Paris-Descartes, and Assistance Publique Hôpitaux de Paris. This work was also supported by the EU framework 6 integrated project MolPAGE (LSHG-CT-2004-512066).

1 Reik W, Dean W, Walter J: Epigenetic reprogramming in mammalian development. Science 2001; 293: 1089-1092.

2 Brandeis M, Ariel M, Cedar H: Dynamics of DNA methylation during development. Bioessays 1993; 11: 709-713.

3 Constancia M, Pickard B, Kelsey G, Reik W: Imprinting mechanisms. Genome Res 1998; 8: 881-900.
4 La Salle S, Mertineit C, Taketo T et al: Windows for sex-specific methylation marked by DNA methyltransferase expression profiles in mouse germ cells. Dev Biol 2004; 268 : 403-415.

5 Kerjean A, Dupont JM, Vasseur C et al: Establishment of the paternal methylation imprint of the human $\mathrm{H} 19$ and MEST/PEG1 genes during spermatogenesis. Hum Mol Genet 2000; 9: 2183-2187.

6 Marques CJ, Carvalho F, Sousa M, Barros A: Genomic imprinting in disruptive spermatogenesis. Lancet 2004; 363: 1700-1726.

7 Marques CJ, Costa P, Vaz B et al: Abnormal methylation of imprinted genes in human sperm is associated with oligozoospermia. Mol Hum Reprod 2008; 14: 67-74.

8 Kobayashi $\mathrm{H}$, Sato A, Otsu $\mathrm{E}$ et al: Aberrant DNA methylation of imprinted loci in sperm from oligospermic patients. Hum Mol Genet 2007; 16: 2542-2512.

9 Tost J, Gut IG: DNA methylation analysis by pyrosequencing. Nat Protoc 2007; 2 : 2265-2275.

10 WHO: World Health Organization Laboratory Manual for the Examination of Human Semen and Sperm-Cervical Mucus Interaction, 4th edn Cambridge, UK: Cambridge University Press, 1999.

11 David G, Bisson JP, Czyglik F et al: Anomalies morphologiques du spermatozoïde humain. (1) Propositions pour un système de classification. J Gynecol Obstet Biol Reprod 1975; (Suppl 1): 17-36.

12 Feil R, Walter J, Allen ND, Reik W: Developmental control of allelic methylation in the imprinted mouse IGF2 and H19 genes. Development 1994; 120: 2933-2943.

13 Lopes S, Lewis A, Hajkova P et al: Epigenetic modifications in an imprinting cluster are controlled by a hierarchy of DMRs suggesting long-range chromatin interactions. Hum Mol Genet 2003; 12: 295-305.

14 Engel N, Raval AK, Thorvaldsen JL, Bartolomei SM: Three-dimentional conformation at the $\mathrm{H} 19$ /lgf2 locus supports a model of enhancer tracking. Hum Mol Genet 2008; 17: 3021-3029.

15 Sullivan MJ, Taniguchi T, Jhee A, Kerr N, Reeve AE: Relaxation of IGF2 imprinting in Wilms' tumours associated with specific changes in IGF2 methylation. Oncogene 1999; 18: 7527-7534.

16 Cui $\mathrm{H}$, Onyango P, Brandenburg S et al: Loss of imprinting in colorectal cancer linked to hypomethylation of H19 and IGF2. Cancer Res 2002; 62: 6442-6446.

17 Cui $\mathrm{H}$, Cruz-Correa M, Giardiello FM et al: Loss of IGF2 imprinting: a potential marker of colorectal cancer risk. Science 2003; 299: 1753-1755.

18 Moore T, Constancia M, Zubair M et al: Multiple imprinted sense and antisense transcripts, differential methylation and tandem repeats in a putative imprinting control region upstream of mouse Igf2. Proc Natl Acad Sci USA 1997; 94: 12509-12514.

19 Monk D, Sanches R, Arnaud P et al: Imprinting of IGF2 PO transcript and novel alternatively spliced INS-IGF2 isoforms show differences between mouse and human. Hum Mol Genet 2006; 15: 1259-1269.

20 Murrell A, Ito Y, Verde G et al: Distinct methylation changes at the IGF2-H19 locus in congenital growth disorders and cancer. PLOS ONE 2008; 3: e1849.

21 Houshdaran S, Cortessis VK, Siegmund K et al: Widespread epigenetic abnormalities suggest a broad DNA methylation erasure defect in abnormal human sperm. PLoS ONE. 2007; 2: e1289.

22 Wu TF, Chu DS: Sperm Chromatin. Fertile grounds for proteomic discovery of clinical tools. Mol Cell Proteomics 2008; 7: 1876-1886.

23 Gatewood JM, Cook GR, Balhorn R, Bradbury EM, Schmid CW: Sequence-specific packaging of DNA in human sperm chromatin. Science 1987; 236: 962-964.

24 Wykes SM, Krawetz SA: The structural organization of sperm chromatin. J Biol Chem 2003; 278: 29471-29477.

25 Banerjee S, Smallwood A: Chromatin modification of imprinted $\mathrm{H} 19$ gene in mammalian spermatozoa. Mol Reprod Dev 1998; 50: 474-484.

26 Bourc'his D, Bestor TH: Meiotic catastrophe and retrotransposon reactivation in male germ cells lacking Dnmt3L. Nature 2004; 431: 96-99.

27 Kaneda M, Okano M, Hata $\mathrm{K}$ et al: Essential role for de novo DNA methyltransferase Dnmt3a in paternal and maternal imprinting. Nature 2004; 429: 900-903.

28 DeBaum MR, Niemitz EL, Feinberg AP: Association of in vitro fertilization with Beckwith-Wiedemann syndrome and epigenetic alterations of LITI and H19. Am J Hum Genet 2003; 72: 156-160.

29 Maher ER, Brueton LA, Bowdin SC et al: Beckwith-Wiedemann syndrome and assisted reproduction technology (ART). J Med Genet 2003; 40: 62-64.

30 Gicquel C, Gaston V, Mandelbaum J et al: In vitro fertilization may increase the risk of Beckwith-Wiedemann syndrome related to the abnormal imprinting of the KCN1OT gene. Am J Hum Genet 2003; 72: 1338-1341.

31 Benchaib M, Ajina M, Lornage J et al: Quantitation by image analysis of global DNA methylation in human spermatozoa and its prognostic value in in vitro fertilization: a preliminary study. Fertil Steril 2003; 80: 947-953.

32 Benchaib M, Braun V, Ressnikof D et al: Influence of global sperm DNA methylation on IVF results. Hum Reprod 2005; 20: 768-773. 